Tropical Journal of Pharmaceutical Research September 2014; 13 (9): 1495-1501

ISSN: $1596-5996$ (print); 1596-9827 (electronic)

(c) Pharmacotherapy Group, Faculty of Pharmacy, University of Benin, Benin City, 300001 Nigeria.

All rights reserved.

Available online at http://www.tjpr.org

Original Research Article

http://dx.doi.org/10.4314/tjpr.v13i9.16

\title{
Screening for Anticandidal and Antibiofilm Activity of Some Herbs in Thailand
}

\author{
Pajaree Kawsud ${ }^{1}$, Jindaporn Puripattanavong ${ }^{2}$ and Rawee Teanpaisan ${ }^{1 *}$ \\ ${ }^{1}$ Common Oral Diseases and Epidemiology Research Center and the Department of Stomatology, Faculty of Dentistry, \\ ${ }^{2}$ Department of Pharmacognosy and Pharmaceutical Botany, Faculty of Pharmaceutical Sciences, Prince of Songkla University, \\ Hat-Yai, 90112, Thailand
}

*For correspondence: Email: rawee.t@psu.ac.th; Tel: +66 74 429878; Fax: +66 74212922

Received: 14 May 2014

Revised accepted: 10 August 2014

\begin{abstract}
Purpose: To evaluate the anticandidal activity of the ethanol extracts of 12 herbs from Thailand. Methods: The herbs studied were Alpinia galanga, Curcuma longa, Curcuma zedoaria, Mentha cordifolia, Ocimum africanum, Ocimum basilicum, Ocimum sanctum, Piper betle, Piper chaba, Piper nigrum, Piper sarmentosum and Zingiber officinale. Various Candida spp. were examined for minimal inhibitory concentration (MIC) and minimal fungicidal concentration (MFC) using microdilution method; time-kill assay was also used to assess the plants. Antibiofilm activity was investigated using a 3-[4, 5dimethyl-2-thiazolyl]-2, 5-diphenyl-2H-tetrazolium-bromide (MTT assay). Gas chromatography mass spectrometry (GC-MS) analysis, thin layer chromatography (TLC) fingerprinting and TLC-bioautography were used to determine the active anticandidal compounds.

Results: All tested herbs, except extracts of $P$. nigrum and Limiaceae family, showed varying zones of inhibition against Candida albicans ATCC 90028. P. betle revealed the strongest anticandidal activity against all tested strains with MIC ranging from 1.56 to $3.13 \mathrm{mg} / \mathrm{ml}$, and MFC from 3.13 to $8.33 \mathrm{mg} / \mathrm{ml}$. Killing activity depended on time and concentrations of the extract. The concentration of $P$. betle extract required to inhibit $\geq 90 \%$ biofilm formation of C. albicans ATCC 90028 was $3.13 \pm 0.15 \mathrm{mg} / \mathrm{ml}$, while that to remove $\geq 90 \%$ biofilm growth was $12.50 \pm 0.69 \mathrm{mg} / \mathrm{ml}$. The result of GC-MS analysis showed the major compound of $P$. betle extract responsible for anticandidal activity as 4-chromanol.

Conclusion: P. betle extract contains 4-chromanol which is a good potential anticandidal agent for the treatment of oral infectious diseases caused by certain Candida spp.
\end{abstract}

Keywords: Piper betle, 4-Chromanol, Anticandida, Biofilm, Candidiasis, Herbs

Tropical Journal of Pharmaceutical Research is indexed by Science Citation Index (SciSearch), Scopus, International Pharmaceutical Abstract, Chemical Abstracts, Embase, Index Copernicus, EBSCO, African Index Medicus, JournalSeek, Journal Citation Reports/Science Edition, Directory of Open Access Journals (DOAJ), African Journal Online, Bioline International, Open-J-Gate and Pharmacy Abstracts

\section{INTRODUCTION}

The genus Candida contains more than 150 species, however, only a limited number of these species regularly cause diseases in humans [1]. Candida species belong to the normal microbiota of human mucosal surfaces including the oral cavity. There has been a significant increase in incidence of oral candidiasis due to higher numbers of immunocompromised patients, the use of dental devices and the use of broad spectrum antifungal drugs. Candida albicans is the main cause of oral candidiasis, however, non-C. albicans (NCAC) species such as Candida glabrata, Candida krusei and Candida tropicalis are now frequently reported as human pathogens [2].

The pathogenesis of oral candidiasis is facilitated by a number of factors including the ability to 
adhere to medical devices and/or host cells, and to form biofilm. Biofilm formation is an important virulence factor for a number of Candida species as it confers significant resistance to antifungal therapy by limiting their penetration through the matrix and protecting cells from host immune responses [3]. It has been demonstrated that biofilms formed by $C$. albicans and/or NCAC strains have been associated with high morbidity and mortality rates [4].

Chlorhexidine $(\mathrm{CHX})$ has been used as an adjunctive therapeutic option for topical use owing to its broad-spectrum antimicrobial efficacy in oral candidiasis. However, it has been known to have side effects with prolonged use $[5,6]$.

Natural products have increasingly received attentions due to their antimicrobial activity against a wide range of human pathogens. Few studies have reported anticandidal activity from various herbs $[7,8]$. In addition, the information on their active compounds is scarce. The aim of this study was to evaluate of the ethanolic extracts of 12 herbs, which are traditionally used in Thai folk medicine against various Candida spp. The phytochemical profile of the most effective extract was also investigated.

\section{EXPERIMENTAL}

\section{Preparation of medicinal herb extracts}

Twelve herbs included in this study were purchased from the local market, and their details are shown in Table 1. The herbs were identified by Dr. Supreeya Yuenyongsawad; Department of Pharmacognosy and Pharmaceutical Botany, Faculty of Pharmaceutical Sciences, Prince of Songkla University, Songkhla, Thailand, where voucher specimens (Table 1) were deposited in the herbarium. The dried plants were macerated with ethanol for 3 days and then filtered through Whatman no. 4 filter paper. The filtrate, each case, was concentrated using a rotary evaporator at $40{ }^{\circ} \mathrm{C}$ and kept at $-20{ }^{\circ} \mathrm{C}$. A $10 \%$ (w/v) stock solution in $10 \%$ dimethyl sulfoxide (DMSO) of each extract was used in this study.

\section{Candida strains and growth conditions}

A total of eleven Candida spp. were tested, and the details of strains used shown in Table 2. In this study, all tested strains were cultured on Sabouraud Dextrose Agar (SDA; Difco Laboratories, Detroit, MI, USA) or Sabouraud Dextrose broth (SDB; Difco Laboratories, Detroit, $\mathrm{MI}, \mathrm{USA})$ at $37^{\circ} \mathrm{C}$ for $24 \mathrm{~h}$.

\section{Antifungal assay}

\section{Agar diffusion test}

A $100 \mu$ l of inoculum $\left(10^{7} \mathrm{cfu} / \mathrm{ml}\right)$ was mixed with $20 \mathrm{ml}$ of warm SDA, and poured into the plate with a $6 \mathrm{~mm}$ diameter metal cup. After solidifying the SDA, the metal cups were removed and the well filled with $100 \mu \mathrm{l}$ of each extract. A $10 \%$ DMSO was included as control. Inhibitory activity was determined by measuring the diameter of the inhibited zone. All tests were performed in triplicates.

\section{Broth microdilution test}

Minimum inhibitory concentration (MIC) and minimum fungicidal concentration (MFC) were determined using the broth microdilution method of the Clinical and Laboratory Standards Institute [9]. Each extract was subjected to a two-fold serial dilutions in the SDB to obtain concentrations from $0.02 \mathrm{mg} / \mathrm{ml}$ to $25 \mathrm{mg} / \mathrm{ml}$ at a total volume of $100 \mu \mathrm{l}$ per well in 96 -well plate, and $100 \mu \mathrm{l}$ of each tested strain $\left(1 \times 10^{3} \mathrm{cfu} / \mathrm{ml}\right)$ was added. The medium as well as $0.1 \% \mathrm{CHX}$ and $10 \%$ DMSO were used as blank, positive control and non-treated control, respectively. MIC was defined as the lowest concentration of the extract that showed no detectable growth. MFC was defined as the lowest concentration of wells that did not grow when $10 \mu \mathrm{l}$ of the well contents was plated on agar. All experiments were repeated thrice in duplicates.

\section{Time-kill assay}

As the screening of extracts revealed that $P$. betle extract gave the strongest anticandidal activity, it was used for the time-kill assay [10]. Growing culture $\left(10^{6} \mathrm{cfu} / \mathrm{ml}\right)$ of representative $C$. albicans ATCC 90028 was added to SDB and exposed to 1,2 and $4 \times$ the MIC of extract, and extract free medium was used as growth control. Samples were removed for colony counts at 0 , $0.5,1,2,4,6,8$, and $24 \mathrm{~h}$. The viable counts were determined using the serial dilution method after incubation. The procedure was repeated in triplicate, and the $\log _{10} \mathrm{cfu} / \mathrm{ml}$ was plotted against time. A $0.1 \% \mathrm{CHX}$ was used as positive control. All assays were carried out in triplicates.

\section{Antibiofilm assay}

\section{Inhibition of biofilm formation}

The effect of $P$. betle extract on biofilm formation of representative C. albicans ATCC 90028 was examined using the modified microdilution method [11]. Two-fold serial dilutions of extract 
and a cell suspension of the tested strain were prepared as described in the MIC assay. A $0.1 \%$ $\mathrm{CHX}$ was used as the positive control. The phosphate buffered saline (PBS) at $\mathrm{pH} 7.0$ and the medium alone were used as the non-treated and blank controls, respectively. After overnight incubation, supernatants were discarded and washed 3 times with PBS. Biofilm formation was quantified using a 3-[4, 5-dimethyl-2-thiazolyl]-2, 5-diphenyl-2H-tetrazolium-bromide (MTT; SigmaAldrich, St Louis, MO, USA) assay. The numbers of surviving Candida were determined by measuring their ability to reduce the yellow tetrazolium salt to a purple formazan product at OD $570 \mathrm{~nm}$. Percentage inhibition was calculated by equation 1 .

$1 \%=\left[1-\left(A_{570}\right.\right.$ of the test $/ A_{570}$ of non-treated control) $\times 100]$

The biofilm inhibition concentration $\mathrm{MBIC}_{50}$ and $\mathrm{MBIC}_{90}$ ) was defined as the concentrations that showed $50 \%$ and $90 \%$ inhibition of biofilm formation. All experiments were repeated in triplicate [11].

\section{Eradication of biofilm formation}

The eradication of biofilm was also examined using minimum biofilm eradication concentration (MBEC) assay. A $200 \mu \mathrm{l}\left(10_{3} \mathrm{cfu} / \mathrm{ml}\right)$ aliquot of $C$. albicans ATCC 90028 was inoculated into 96well plates to form biofilm overnight. The biofilm was washed 3 times with PBS. The biofilm was exposed to a $200 \mu \mathrm{l}$ of various concentrations of extract ranged from 0.02 to $25 \mathrm{mg} / \mathrm{ml}$. The number of surviving Candida were determined using MTT assay at OD $570 \mathrm{~nm}$. Percentage eradication was calculated by equation 2 .

$E \%=\left[1-\left(A_{570}\right.\right.$ of the test $/ A_{570}$ of non-treated control) $\times 100]$

MBEC value was defined as the concentrations that showed 50 and $90 \%$ eradication of biofilm formation. The medium alone, $0.1 \% \mathrm{CHX}$ and PBS were used as the blank control, positive and non-treated, respectively. All experiments were repeated thrice in duplicate [11].

GC-MS analysis of phytoconstituents in $P$. betle extract

One microliter of $P$. betle extract was investigated for its phytoconstituents using gas chromatography-mass spectrometer (GC-MS, Trace GC Ultra, USA), the TR-5MS capillary column $(0.25 \mathrm{~mm} \times 30 \mathrm{~m} \times 0.25 \mu \mathrm{m}$ film thickness) was used for the separation of various phytoconstituents. The GC conditions were: injection temperature, $250{ }^{\circ} \mathrm{C}$; column temperature, isothermal at $70{ }^{\circ} \mathrm{C}$ for $2 \mathrm{~min}$, then programmed to $305^{\circ} \mathrm{C}$ at $10{ }^{\circ} \mathrm{C} / \mathrm{min}$ and held at this temperature for $5 \mathrm{~min}$. Helium gas with a flow rate of $1.0 \mathrm{ml} / \mathrm{min}$ was used as a carrier gas. The effluent of the GC column was introduced directly into the source of MS and spectra obtained in the electron ionization mode with 70 eV ionization energy, the sector mass analyzer was set to scan between 35-500 amu for 2 s. The identification of individual unknown compound is based on comparing its retention time relative to those of the known components by matching spectral peaks available with Wiley 9 Mass Spectral Library [12].

\section{Thin layer chromatography (TLC) fingerprinting of $P$. betle and TLC- bioautography-assisted separation of anticandidal}

For TLC-fingerprinting study, $P$. betle extract (100 mg) and pure eugenol $(5 \mu \mathrm{l})$ were prepared in $1 \mathrm{ml}$ of $95 \%$ ethanol. A $10 \mu \mathrm{l}$ of each sample was applied onto the alumina silica gel $\mathrm{GF}_{254}$ TLC-plate, and was run through a series of solvent systems using a mixture of 90 volumes of toluene and 10 volume of ethyl acetate as the mobile phase. The plates were dried in the air, and were stained with a $20 \% \quad(w / v)$ of phosphomolybdic acid in ethanol [13].

TLC-bioautography was performed using agar overlay method. TLC-plates were placed on plate contained $6 \mathrm{ml}$ of SDA, and $10 \mathrm{ml}$ of top agar with $100 \mu$ of inoculum $\left(10^{7} \mathrm{cfu} / \mathrm{ml}\right)$ was covered on TLC plate. After overnight incubation, the inhibited zone was observed as clear zone. The relative front values $(\mathrm{Rf})$ was calculated as: $\mathrm{Rf}=$ distance travelled by solute / distance travelled by solvent [14].

\section{Statistical analysis}

The data are expressed as mean \pm standard deviation (SD) by using Microsoft Excel 2007 12.0.6611.1000 (Microsoft Corporation, Redmond, WA, USA) from the three experiments with duplicate independent experiments. The graph of time-kill analysis is plotted using SigmaPlot Version 11.0 (Systat Software, Inc., San Jose, CA, USA).

\section{RESULTS}

Anticandidal activity of 12 herbs against $C$. albicans was shown in Table 1. These extracts showed different inhibited zone against $C$. albicans ATCC 90028. The diameter of inhibited zone for all the extracts ranged from 1 to $20 \mathrm{~mm}$, 
except $P$. nigrum and extracts of Lamiaceae did not show any inhibitory zone. Two herbs $P$. betle and $C$. zedoaria, revealed the good anticandidal activity, and were further examined for their MIC and MFC against 11 Candida strains. $P$. betle exhibited a better anticandidal activity than $C$. zedoaria against all tested strains with the MICs ranged from 1.56 to $3.13 \mathrm{mg} / \mathrm{ml}$ and MFCs ranged from 3.13 to $8.33 \mathrm{mg} / \mathrm{ml}$, respectively (Table 2).

Time kill assay of $P$. betle extract was performed for $C$. albicans ATCC 90028, it was depended on time and concentrations of extract. At $1 \times \mathrm{MIC}, 2$ $\times$ MIC and $4 \times$ MIC, the strain was killed after 8 , 2 and $1 \mathrm{~h}$, respectively. The killing of $0.1 \% \mathrm{CHX}$ was observed within $30 \mathrm{~min}$ (Figure 1). The concentrations of $P$. betle extract required to inhibit $\geq 50 \%$ and $\geq 90 \%$ biofilm formation of $C$. albicans ATCC 90028 were $0.78 \pm 0.31 \mathrm{mg} / \mathrm{ml}$ and $3.13 \pm 0.15 \mathrm{mg} / \mathrm{ml}$, respectively. The concentrations of $P$. betle extract to remove of $\geq$ $50 \%$ and $\geq 90 \%$ biofilm formation were $1.56 \pm$ $0.44 \mathrm{mg} / \mathrm{ml}$ and $12.5 \pm 0.69 \mathrm{mg} / \mathrm{ml}$, respectively. $\mathrm{CHX}$ could completely inhibit or remove biofilm formation (Figure 2). The GC-MS analysis of $P$. betle extract showed 2 major chemical components of 4-chromanol $(62.33 \%)$ and eugenol $(17.10 \%)$, which their retention times were 10.46 and 9.11 , respectively (Figure $3 \mathrm{~A}$ ). It was demonstrated that there were several bands of components appeared on the TLC-plate (Figure $3 \mathrm{~B}$ ), and one major band with $\mathrm{Rf}$ at 0.38 gave the large inhibited clear zone against tested Candida in TLC-bioautography assay (Figure 3C). Another minor band with $\mathrm{Rf}$ at 0.74 matched to the standard eugenol did not show any inhibited zone.

Table 1: Plants and susceptibility of Candida albicans ATCC 90028

\begin{tabular}{|c|c|c|c|c|}
\hline Family & Scientific name & $\begin{array}{c}\text { Voucher specimen } \\
\text { no. }\end{array}$ & Used part & $\begin{array}{l}\text { Anticandidal } \\
\text { activity }\end{array}$ \\
\hline \multirow[t]{4}{*}{ Lamiaceae } & Mentha cordifolia Opiz. & SKP 095130301 & Leaf & - \\
\hline & Ocimum basilicum $\mathrm{L}$. & SKP 095150201 & Aerial parts & - \\
\hline & Ocimum africanum Lour. & SKP 095150101 & Aerial parts & - \\
\hline & Ocimum sanctum $\mathrm{L}$. & SKP 095151901 & Aerial parts & - \\
\hline \multirow[t]{4}{*}{ Piperaceae } & Piper betle L. & SKP 146160201 & Leaf & +++ \\
\hline & Piper chaba Hunter & SKP 146160301 & Fruit & + \\
\hline & Piper nigrum L. & SKP 146161401 & Fruit & - \\
\hline & Piper sarmentosum Roxb. & SKP 146161906 & Leaf & + \\
\hline \multirow[t]{4}{*}{ Zingiberaceae } & Alpinia galanga (L.) Willd. & SKP 206010701 & Rhizome & + \\
\hline & Curcuma longa L. & SKP 206031201 & Rhizome & + \\
\hline & Curcuma zedoaria Roscoe & SKP 206032601 & Rhizome & ++ \\
\hline & Zingiber officinale Roscoe & SKP 206261501 & Rhizomes & + \\
\hline
\end{tabular}

+, $0<$ zone < $10 \mathrm{~mm}$; + +, $10 \mathrm{~mm} \leq$ zone < $20 \mathrm{~mm}$; + + +, $20 \mathrm{~mm} \leq$ zone; -, absence of inhibition zone

Table 2: MIC and MFC of tested extracts against Candida spp

\begin{tabular}{lcccc}
\hline \multirow{2}{*}{ Strain } & \multicolumn{3}{c}{ Concentration of extracts (mg/ml) } \\
\cline { 2 - 5 } & \multicolumn{2}{c}{ Curcuma zedoaria } & \multicolumn{2}{c}{ Piper betle } \\
\cline { 2 - 4 } & MIC & MFC & MIC & MFC \\
\hline Candida albicans ATCC 10231 & $2.6 \pm 0.9$ & $>25$ & $3.13 \pm 0$ & $4.17 \pm 1.8$ \\
Candida albicans ATCC 90028 & $2.6 \pm 0.9$ & $>25$ & $3.13 \pm 0$ & $3.13 \pm 0$ \\
Candida dubliniensis MYA-577 & $2.6 \pm 0.9$ & $25 \pm 0$ & $2.60 \pm 0.9$ & $4.17 \pm 1.8$ \\
Candida dubliniensis MYA-646 & $2.6 \pm 0.9$ & $25 \pm 0$ & $2.08 \pm 0.9$ & $3.13 \pm 0$ \\
Candida glabrata ATTC 66032 & $6.25 \pm 0$ & $>25$ & $3.13 \pm 0$ & $6.25 \pm 0$ \\
Candida glabrata ATTC 90030 & $6.25 \pm 0$ & $>25$ & $2.60 \pm 0.9$ & $5.21 \pm 1.8$ \\
Candida krusei ATTC 6258 & $8.33 \pm 3.6$ & $>25$ & $3.13 \pm 0$ & $8.33 \pm 3.6$ \\
Candida krusei ATTC 34135 & $8.33 \pm 3.6$ & $>25$ & $3.13 \pm 0$ & $6.25 \pm 0$ \\
Candida tropicalis ATTC 750 & $2.60 \pm 0.9$ & $>25$ & $2.60 \pm 0.9$ & $4.17 \pm 1.8$ \\
Candida tropicalis ATTC 13803 & $2.60 \pm 0.9$ & $>25$ & $2.08 \pm 0.9$ & $3.13 \pm 0$ \\
Candida tropicalis ATTC 66029 & $1.56 \pm 0$ & $25 \pm 0$ & $1.56 \pm 0$ & $4.17 \pm 1.8$ \\
\hline
\end{tabular}

MIC - the minimum inhibitory concentration; MFC - the minimum fungicidal concentration; the values represent data obtained from three independent assays and where the appropriate mean \pm S.D. is given 




Figure 1: Time-kill curves of $C$. albicans ATCC 90028 at different concentrations of $P$. betle extract: $0 \times \mathrm{MIC}$ $(\triangle), 1 \times \operatorname{MIC}(\nabla), 2 \times \operatorname{MIC}(\bigcirc)$ and $4 \times \operatorname{MIC}(\bullet) ; 0.1 \%$ $\mathrm{CHX}(\boldsymbol{\square})$. Each symbol indicated the mean $\pm \mathrm{SD}$

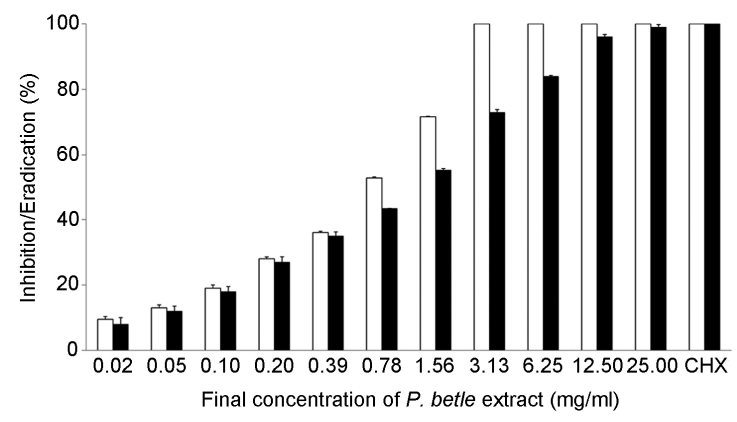

Figure 2: Inhibition ( $\square$ ) and eradication ( $\boldsymbol{\square}$ ) of biofilm of $C$. albicans ATCC 90028 by $P$. betle extract at various concentrations; $0.1 \%(\mathrm{w} / \mathrm{v}) \mathrm{CHX}$ was used as control. Error bars indicate standard deviations; $n=6$

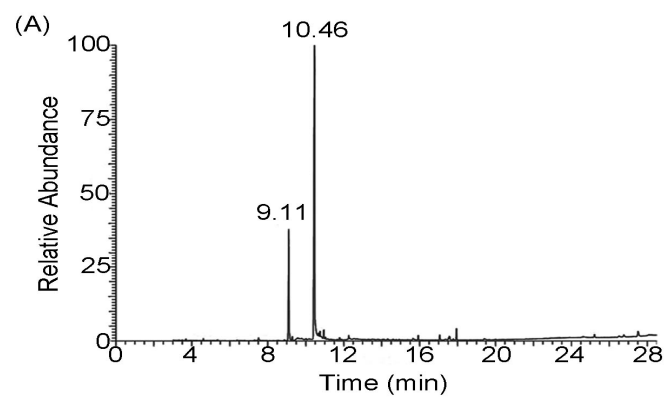

(B)

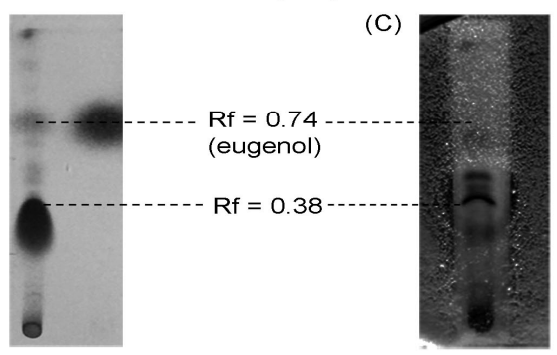

Figure 3: GC-MS chromatogram (A), TLC fingerprinting (B) and TLC-bioautography against $C$. albicans ATCC 90028 (C) of $P$. betle leaves extract

\section{DISCUSSION}

Candida species are the most common fungal pathogens of humans and are the causative agents at various locations in the body, giving rise to severe morbidity in millions of individuals worldwide [15]. In oral cavity, C. albicans is the organism most frequently implicated in a range of oral candidiasis like oropharyngeal candidiasis, oral thrush and denture stomatitis [15], and NCAC have been found dramatically in oral candidal infection. A topical use of $\mathrm{CHX}$ mouthwash is the most common antimicrobial substance to control oral candidiasis. However, $\mathrm{CHX}$ has been reported as having many unwanted effects including taste disturbance, desquamative lesions, and brown staining of the teeth and oral mucosa [5,6] and having limited use in the pregnant and the young children.

Medicinal herbs are widely employed in folk medicine, mainly in communities with low hygiene and poor sanitation. Plants products are considered to have low side effects compared with synthetic ones. Some studies have demonstrated that herbs possess the anticandidal activity against various Candida strains $[7,8]$. In this study, 7 of 12 Thai herbs, $A$. galanga, $C$. longa, $C$. zedoaria, $P$. betle, $P$. chaba, $P$. sarmentosum and $Z$. officinale showed potential anticandidal activity against tested $C$. albicans. However, P. nigrum and plant extracts in family of Lamiaceae did not any activity possible because their major potent compounds are volatile oil which could have evaporated during preparation of extracts by a rotary evaporator. Among those plants tested, $P$. betle extract exhibited the strongest anticandidal activity against tested Candida species.

In addition, $P$. betle extract has shown an antibiofilm activity. Biofilm of $C$. albicans and NCAC are associated with high incidence of hospital morbidity and mortality [4] due to the increased use of medical devices such as lens, implants and dentures. Such biomaterials facilitate Candida strains to colonize and form biofilm leading to the development of fungal resistance to antifugal agents such as amphotericin B, ketoconazole and fluconazole [16]. Experiments comparing biofilm of Candida in this study have demonstrated that higher concentrations of $\mathrm{MBEC}_{90}$ of $P$. betle extract (4 folds) than of $\mathrm{MBIC}_{90}$ are required to significantly remove existing biofilm cells. This is an expected result since Candida in the biofilm is strongly protected and less susceptible to antifungal 
agents than in planktonic form [17]. It was shown that $P$. betle extract was able to remove Candida in the dose and time dependent manner. Our results indicated that $P$. betle extract acts as a potent antibiofilm agent that has dual actions of preventing biofilm formation and removing existing biofilm.

$P$. betle has been extensively used in traditional medicines in many countries. It was reported to have various pharmacological activities such as antifungal, antimicrobial, immunomodulatory and anti-inflammatory [18].

The antimicrobial activity has been previously shown to be a broad spectrum of both bacteriostatic and fungistatic effects against $C$. albicans, Pseudomonas aeruginosa, Aspergillus flavus and Escherichia coli $[19,20]$. Some studies reported an anticandidal effect of $P$. betle on a wide range of Candida spp. [7,20]. Our results are in concordance with the study of HimratulAznita et al [20] who demonstrated the anticandidal activity of aqueous crude extract of $P$. betle leaves against 7 oral Candida species. However, there was no information of antibiofilm or active compounds mentioned in that study. The ethanol extract in this study revealed the lower MICs and MFCs of anticandidal activity compared to the aqueous extract [20]. This was also found in a study of the ethanol extract of $P$. betle leaves with stronger antibacterial activity compared with aqueous extract [21]. It indicates that the ethanol may be the appropriate solvent for $P$. betle leaves extraction.

The results of the GC-MS analysis, TLC fingerprinting and TLC-bioautography in the present study indicated that 4-chromanol could be the active compound responsible for the anticandidal activity. There were only 2 major peaks found in the GC-MS analysis, and the one with the lower peak matched to the eugenol by the TLC fingerprinting. Eugenol did not exhibit any anticandidal activity in TLC-bioautography assay. Therefore, the major band found on the TLC fingerprinting with $\mathrm{Rf}$ of 0.38 showing a large inhibited zone against Candida strain should be the highest peak of 4-chromanol found in GC-MS analysis.

A number of phytochemical constituents of $P$. betle extract have been documented in some studies [18,22]; however, 4-chromanol has not be reported. The finding of the phytochemical profile of $P$. betle extract in this study is in agreement with Deshpande and Kadam [21], in which the extract revealed the presence of 4- chromanol. However, the presence of 4chromanol was a major component in this study $(62.33 \%)$, which was higher than earlier reported that was higher than their report. The finding of chemotypic variations in this study and others may be due to ecological and geographical conditions, age of herb and time of harvesting. The apparent variation in the chemical profile may influence their anticandidal activity.

\section{CONCLUSION}

The current study supports the traditional uses of the studied plant and suggests that the $P$. betle leaves extract is a potential source of natural anticandidal agent. It possesses 4-chromanol with good anticandidal properties that may be used for oral infectious diseases caused by certain Candida spp. Further work should be performed to determine its mode of action as well as in vivo activity.

\section{ACKNOWLEDGEMENT}

This work was supported by the Higher Education Research Promotion and National Research University Project of Thailand, Office of the Higher Education Commission (DEN5405475).

\section{REFERENCES}

1. Scully C, El-Kabir M, Samaranayake LP. Candida and oral candidosis: a review. Crit Rev Oral Biol Med 1994; 5(2): 125-157.

2. Thompson GR 3rd, Patel PK, Kirkpatrick WR, Westbrook SD, Berg D, Erlandsen J, Redding SW, Patterson TF. Oropharyngeal candidiasis in the era of antiretroviral therapy. Oral Surg Oral Med Oral Pathol Oral Radiol Endod 2010; 109(4): 488-495.

3. Ramage G, Saville SP, Thomas DP, López-Ribot JL. Candida biofilms: an update. Eukaryot Cell 2005; 4(4): 633-638.

4. Kumamoto CA. Candida biofilms. Curr Opin Microbiol 2002; 5(6): 608-611.

5. Addy M, Moran J. Mechanisms of stain formation on teeth, in particular associated with metal ions and antiseptics. Adv Dent Res 1995; 9(4): 450-456.

6. Flötra L, Gjermo P, Rölla G, Waerhaug J. Side effect of chlorhexidine mouthwashes. Scand J Dent Res 1971; 79(2): 119-125.

7. Nazmul MHM, Salmah I, Syahid A, Mahmood AA. In-vitro screening of antifungal activity of plants in Malaysia. Biomed Res 2011; 22(1): 28-30.

8. Rukayadi Y, Shim JS, Hwang JK. Screening of Thai medicinal plants for anticandidal activity. Mycoses 2008; 51(4): 308-312. 
9. [CLSI] Clinical Laboratory Standards Institute. Reference method for broth dilution Antifungal susceptibility testing of yeasts. Approved Standard M27-A2. Wayne, PA: Clinical Laboratory Standards Institute, 2002.

10. Klepser ME, Wolfe EJ, Jones RN, Nightingale $\mathrm{CH}$, Pfaller MA. Antifungal pharmacodynamic characteristics of fluconazole and amphotericin $B$ tested against Candida albicans. Antimicrob Agents Chemother 1997; 41(6): 1392-1395.

11. Tang HJ, Chen CC, Ko WC, Yu WL, Chiang SR, Chuang $Y C$. In vitro efficacy of antimicrobial agents against high-inoculum or biofilm embedded methicillinresistant Staphylococcus aureus with vancomycin minimal inhibitory concentrations equal to $2 \mu \mathrm{g} / \mathrm{ml}$ (VA2-MRSA). Int J Antimicrob Agents. 2011; 38(1): 46-51.

12. Annegowda HV, Tan PY, Mordi MN, Ramanathan S, Hamdan MR, Sulaiman MH, Mansor SM. TLCbioautography-guided isolation, HPTLC and GC-MSassisted analysis of bioactives of Piper betle leaf extract obtained from various extraction techniques: in vitro evaluation of phenolic content, antioxidant and antimicrobial activities. Food Anal Methods 2013; 6(3): 715-726.

13. Department of Medical Sciences, Ministry of Public Health, Thailand. Thai Herbal Pharmacopoeia volume II. Bangkok: Office of Notional Buddishm Press; 2007. pp 63-70.

14. Rahalison L, Hamburger MO, Hostettmann K, Hostettmann K, Monod M, Frenk E. A bioautographic agar overlay method for the detection of antifungal compounds from higher plants. Phytochem Anal 1991; 2(5): 199-208
15. Ruhnke M. Epidemiology of Candida albicans infections and role of non-Candida-albicans yeasts. Curr Drug Targets 2006; 7(4): 495-504.

16. Joly V, Bolard J, Yeni P. In vitro models for studying toxicity of antifungal agents. Antimicrob Agents Chemother 1992; 36(9): 1799-1804.

17. Donlan RM, Costerton JW. Biofilms: survival mechanisms of clinically relevant microorganisms. Clin Microbiol Rev 2002; 15(2): 167-193.

18. Nagori K, Singh MK, Alexander A, Kumar T, Dewangan $D$, Badwaik H, Tripathi DK. Piper betlel: A review on its ethnobotany, phytochemistry, pharmacological profile and profiling by new hyphenated technique DART-MS (Direct Analysis in Real Time Mass Spectrometry). J Pharmacy Res 2011; 4(9): 2991 2997.

19. Chanudom L, Bhoopong P, Khwanchuea R, Tangpong J. Antioxidant and antimicrobial activities of aqueous \& ethanol crude extract of 13 Thai traditional plants. Int J Curr Microbiol App Sci 2014; 3(1): 549-558.

20. Himratul-Aznita WH, Mohd-Al-Faisal N, Fathilah AR. Determination of the percentage inhibition of diameter growth (PIDG) of Piper betle crude aqueous extract against oral Candida species. J Med Plants Res 2011; 5(6): 878-884.

21. Deshpande SN, Kadam DG. GCMS analysis and antibacterial activity of Piper betle (Linn) leaves against Stroptococcus mutans. Asian J Pharm Clin Res 2013; 6(5): 99-101.

22. Prakash B, Shukla $R$, Singh $P$, Kumar A, Mishra PK, Dubey NK. Efficacy of chemically characterized Piper betle L. essential oil against fungal and aflatoxin contamination of some edible commodities and its antioxidant activity. Int J Food Microbiol 2010; 142(12): 114-119. 